\title{
Número e intervalo de pesagens para estimação de parâmetros de curvas de crescimento em bovinos
}

\author{
Fábio Luiz Buranelo Toral ${ }^{1}$
}

1 Universidade Federal de Mato Grosso.

RESUMO - Este trabalho foi realizado com os objetivos de verificar a influência do número e do intervalo de pesagens sobre as estimativas do peso assintótico $(A)$ e da taxa de maturação $(k)$ de bovinos e identificar o número e intervalo mínimos de pesagens para obtenção de estimativas confiáveis para esses parâmetros. Foram simulados valores de $A$ e $k$ do modelo de Richards e pesos de bovinos em idades entre 1 e 3.601 dias e gerados conjuntos de dados com número de pesagens variando de 13 a 121, obtidas a cada 30 dias, além de conjuntos com pesos entre as idades de 1 e 3.601 dias, mas com intervalos de pesagens variando de 30 a 150 dias. A diminuição no número de pesagens utilizadas para estimação de $A$ e $k$ reduziu a correlação entre os valores simulados e estimados e aumentou o viés e a média dos resíduos ao quadrado. O aumento do intervalo de pesagens não influenciou as estimativas de $A$, mas prejudicou as estimativas de $k$. Pesagens a cada 30 dias por um período de quatro anos, a cada 60 dias por cinco anos ou a cada 90 dias por seis anos, pelo menos, são suficientes para obtenção de estimativas confiáveis dos parâmetros $A$ e $k$ do modelo de Richards para bovinos.

Palavras-chave: modelo não-linear, peso assintótico, Richards, taxa de maturação

\section{Number and interval of weightings to estimate parameters of the growth curve of cattle}

\begin{abstract}
This study aimed to evaluate the minimum number and interval of weightings to estimate the asymptotic weight $(A)$ and maturing rate $(k)$ parameters of the growth curve of cattle. Parameters $A$ and $k$ of the Richards non-linear model and two data sets of weights of cattle from 1 to 3,601 days were simulated. The first data set consisted of 13 to 121 weights obtained every 30 days and the second one consisted of weightings at intervals of 30 to 150 days. The decrease in number of weightings used to estimate $A$ and $k$ reduced the correlations between estimated and simulated parameters and increased the bias and the residuals mean square. The increase in the interval of weightings did not affect the estimates of $A$, but had a damaging effect on the estimates of $k$. Weightings every 30 days and over four years, every 60 days and over five years or every 90 days and over six years, at least, are sufficient to estimate the parameters $A$ and $k$ of the Richards non-linear model for cattle.
\end{abstract}

Key Words: asymptotic weight, maturing rate, non-linear models, Richards

\section{Introdução}

Os modelos não-lineares podem ser utilizados na análise de dados de crescimento de bovinos e permitem que parte das informações contidas nas medidas peso-idade seja resumida em menor número de parâmetros com interpretação biológica. O modelo de Richards (1959) tem se mostrado adequado no ajuste de curvas de crescimento de bovinos (Brown et al., 1976; Perotto et al., 1997) e, segundo Perotto et al. (1997), os parâmetros $A$ e $k$ podem ser interpretados como peso na idade adulta e taxa de maturação, respectivamente.

O interesse na estimação do peso adulto e da taxa de maturação de bovinos pode ser explicado por estas carac- terísticas estarem associadas a número e quilogramas de bezerros desmamados por vaca/ano (López de Torre et al., 1992; Barbosa et al., 2002), idade ao primeiro parto, produção de leite, duração da primeira lactação, longevidade (Coelho et al., 2006) e exigência nutricional das vacas (Ferrell \& Jenkins, 1984). Além disso, as herdabilidades de $A$ e $k$ são próximas de 0,40 (Silva et al., 2000; Garnero et al., 2005) e sugerem a possibilidade de alteração do padrão de crescimento por meio da seleção.

Morrow et al. (1978) trabalharam com dados de crescimento de vacas Angus e verificaram alterações nas médias estimadas dos parâmetro s $A$ e $k$ quando a idade máxima de pesagem variou de 2,5 a 8,5 anos ou mais. O número e o 
intervalo das pesagens disponíveis de cada animal também podem influenciar as estimativas de $A$ e $k$ (McLaren et al., 1982). Quando os dados disponíveis contemplam apenas o crescimento antes de os animais atingirem a maturidade, as estimativas de $A$ e $k$ podem ser consideradas extrapolações e estar sujeitas a erros (Garnero et al., 2005).

Freqüentemente os ajustes de modelos não-lineares de crescimento têm sido realizados com conjuntos de dados que não contemplam o crescimento até a maturidade e as inferências sobre os parâmetros estimados podem estar sujeitas a erros. Por outro lado, a utilização de número elevado de medidas peso-idade para cada animal também pode inviabilizar a realização de experimentos, em razão da mão-de-obra e do tempo necessários para obtenção dos dados. Dessa forma, este trabalho foi realizado com os objetivos de verificar as influências do número e do intervalo de pesagens sobre as estimativas dos parâmetros $A$ e $k$ e identificar o número e o intervalo mínimo de pesagens para obtenção de estimativas confiáveis para esses parâmetros, utilizando o modelo não-linear de Richards (1959).

\section{Material e Métodos}

Os pesos utilizados neste trabalho foram simulados utilizando-se o modelo não-linear de Richards (1959):

$$
y_{i j}=A_{i}\left(1-b_{i} e^{-k_{i} T_{j}}\right)^{m_{i}}+\varepsilon_{i j},
$$

em que: $y_{i j}=$ peso do animal $i$ na idade $j ; A_{i}=$ peso assintótico do animal $i ; b_{i}=$ constante de integração para a curva de crescimento do animal $i ; e=$ base do sistema natural de logaritmos; $k_{i}=$ taxa segundo a qual a função logarítmica de $y_{i}$ muda linearmente com o tempo; $T_{j}=$ idade $j$, em dias; $m_{i}=$ variável que determina em que proporção do tamanho final ocorre o ponto de inflexão da curva de crescimento do animal $i$ (Perotto et al., 1997); e $\varepsilon_{i j}=$ resíduo aleatório, associado a cada observação. Foram simulados inicialmente valores de $A_{i} \sim N(441 ; 144), b_{i} \sim N\left(0,523 ; 3,23 \times 10^{-5}\right)$; $m_{i} \sim N\left(3,8416 ; 2,8561 \times 10^{-4}\right) ; \quad$ e $\quad \varepsilon_{i j} \sim N\left(0 ; 0,015 \times \bar{y}_{j}\right)$ para 10.000 animais.

Os valores de $k_{i}$ foram simulados de maneira semelhante àquela utilizada por Clément et al. (2001), ou seja:

$$
k_{i}=\bar{k}+r_{A k} \times \frac{\sigma_{k}}{\sigma_{A}} \times A_{i}+\sqrt{\left(1-r_{A k}^{2}\right)} \times Q_{i} \times \sigma_{k}
$$

em que: $\bar{k}=0,0039645$ representa uma constante presente em todos os valores de $k_{i} ; r_{A k}=-0,75$ a correlação fenotípica entre $A$ e $k ; \sigma_{k}=6,51 \times 10^{-5}$ e $\sigma_{A}=12$ os desvios-padrão de $k$ e $A$, respectivamente; e $Q_{i} \sim N(0 ; 1)$. Após a simulação desses parâmetros, os pesos de cada animal, nas idades de 1 a 3.601 dias, com intervalos de 30 dias, foram obtidos de acordo com o modelo não-linear de Richards (1959), totalizando 121 observações por animal. Optou-se pela utilização de pesos mensais porque há relatos da utilização desse intervalo de pesagem para bovinos (Nobre et al., 1987; Tedeschi et al., 2000; Barbosa et al., 2002; Paz et al., 2004), especialmente em rebanhos experimentais.

Foram simulados dez arquivos de dados (A1, .., A10) como descrito anteriormente e, em seguida, foram formados subarquivos excluindo-se os pesos obtidos em idades superiores a $361(\mathrm{~A} 1 / \mathrm{id} 361, \ldots, \mathrm{A} 10 / \mathrm{id} 361), 721,1.081,1.441$, 1.801 (A1/id1.801, ..., A10/id1.801), 2.161, 2.521, 2.881, 3.241 e 3.601 (A1/id3.601, ..., A10/id3.601) dias, para cada arquivo simulado. Com isso, foram obtidos subarquivos com 13, 25 , $37,49,61,73,85,97,109$ e 121 observações mensais de pesoidade, que representam, aproximadamente, pesagens até 1 , 2, 3, 4, 5, 6, 7, 8, 9 e 10 anos de idade, respectivamente.

Para avaliar a influência do intervalo de pesagens sobre as estimativas de parâmetros, foram formados subarquivos contendo apenas os pesos obtidos a cada 30 (A1/int $30, \ldots$, A10/int30), 60, 90 (A1/int90, ..., A10/int90), 120 e 150 (A1/int150, ..., A10/int150) dias, a partir da idade 1. Dessa forma, foram gerados subarquivos com 121, 61, 41, 31 e 25 observações de peso-idade, obtidas até a idade máxima de 3.601 dias para essas situações.

Os parâmetros do modelo de Richards (1959) foram estimados, para cada animal, utilizando-se o método de Gauss-Newton. As estimativas dos parâmetros $A$ e $k$, obtidas para cada animal, foram comparadas com os valores simulados analisando-se a correlação entre os valores verdadeiros e os estimados $(r)$, o viés (v) e a média dos resíduos ao quadrado $(M R Q)$, obtidos das seguintes formas:

$$
\begin{aligned}
& r=\frac{\sigma_{\hat{p} p}}{\sqrt{\sigma_{\hat{p}}^{2} \times \sigma_{p}^{2}}} ; \\
& v=\frac{\frac{1}{n} \sum_{i=1}^{n} \hat{p}_{i}-p_{i}}{\bar{p}} \times 100 ; \\
& M R Q=\frac{1}{n} \sum_{i=1}^{n}\left(\hat{p}_{i}-p_{i}\right)^{2},
\end{aligned}
$$

em que: $\hat{p}_{i}=$ estimativa do parâmetro $(k$ ou $A)$ do animal $i$; $p_{i}=$ valor simulado para o parâmetro do animal $i$; $\bar{p}=$ média do parâmetro $; \sigma_{\hat{p} p}=$ co-variância entre $\hat{p}_{i}$ e $p_{i}$; $\sigma_{\hat{p}}^{2}$ e $\sigma_{p}^{2}$ as variâncias de $\hat{p}$ e $p$, respectivamente; e $n=$ número de observações para os parâmetros, obtidas após a convergência do algoritmo. Esses critérios foram obtidos para cada uma das repetições e as médias dos dez valores obtidos para cada situação (dez situações quanto à idade na última pesagem e cinco situações quando ao 
intervalo de pesagens) foram utilizadas para apresentação. As análises de simulação, estimação de parâmetros e obtenção de correlações, vieses e médias dos desvios ao quadrado foram realizadas utilizando-se os procedimentos do SAS (SAS, 2002).

\section{Resultados e Discussão}

Os resultados das médias e dos valores mínimos e máximos simulados para os parâmetros $A$ e $k$ e as correlações entre eles (Tabela 1) estão de acordo com a amplitude reportada por Santoro et al. (2005), que utilizaram dados de fêmeas zebuínas criadas em Pernambuco. Para a realização das simulações, considerou-se a correlação entre os parâmetros $A$ e $k$ de $-0,75$, igual ao estimado por Silva et al. (2000) com dados de fêmeas da raça Canchim. A correlação negativa entre estes parâmetros indica que vacas com maior peso assintótico (A) precisam de mais tempo para atingir este peso, em virtude da menor taxa de maturação $(k)$. Por outro lado, vacas com maiores valores de $k$ atingem o peso adulto mais rápido porque este valor geralmente é menor que no primeiro caso.

Os pesos simulados de acordo com a idade (Figura 1) foram próximos aos estimados por Perotto et al. (1997) utilizando o modelo de Richards (1959) para dados de fêmeas Guzerá, Gir, Holandês $\times$ Guzerá e Holandês $\times$ Gir criadas no Paraná. Dessa forma, é possível afirmar que o fenômeno de crescimento de fêmeas bovinas simulado é representativo de valores provenientes de dados reais, demonstrando a viabilidade dessas simulações para o estudo do número e do intervalo mínimo de pesagens para estimação de parâmetros de crescimento do modelo nãolinear de Richards (1959).

Com a utilização de 121 pesagens (Figura 2), que corresponde à utilização de pares de medidas peso-idade

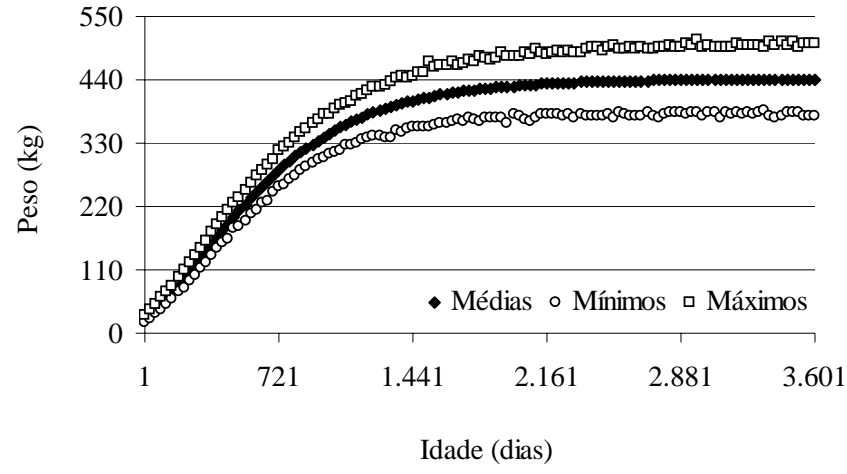

Figura 1 - Médias e valores mínimos e máximos para os pesos simulados nos dez conjuntos de dados, de acordo com a idade.

tomadas do nascimento aos 3.601 dias de idade (9,8 anos), as médias das correlações entre os valores estimados e os simulados foram próximas a 1,00 e 0,91 para $A$ e $k$ do modelo não-linear de Richards (1959), respectivamente. Reduções superiores a 10\% nas correlações entre os valores estimados e os simulados, em relação às correlações obtidas com a utilização de 121 pares de medida peso-idade, foram observadas quando os números de medidas utilizadas foram inferiores a 49 e 61 para $A$ e $k$, respectivamente.

Com a redução do número de pesagens para obtenção das estimativas (Figura 2), os valores de $A$ e $k$ foram cada vez mais super e subestimados, respectivamente. Essas alterações aumentaram com a utilização de menos de 49 medidas. Esses resultados confirmam aqueles obtidos por Morrow et al. (1978), que analisaram os dados trimestrais de peso-idade de vacas Angus paridas aos 3 anos de idade. Esses autores verificaram aumento das médias para o parâmetro $A$ e redução das médias para o parâmetro $k$, com a diminuição do número de pesagens utilizadas para obtenção das estimativas. A diferença no comportamento

Tabela 1 - Médias (Me), valores mínimos (Min) e máximos (Max) para os parâmetros $A$ e $k$ e correlações entre eles ( $r_{A k}$ ) nos arquivos de dados simulados de acordo com o modelo não-linear de Richards (1959)

\begin{tabular}{|c|c|c|c|c|c|c|c|}
\hline \multirow[t]{2}{*}{ Arquivo } & \multicolumn{3}{|c|}{$A(\mathrm{~kg})$} & \multicolumn{3}{|c|}{$k(1 / \mathrm{dia})$} & \multirow[t]{2}{*}{$r_{A k}$} \\
\hline & $\mathrm{Me}$ & Min & Max & $\mathrm{Me}$ & Min & Max & \\
\hline A 1 & 440,87 & 391,26 & 492,09 & 0,00217 & 0,00188 & 0,00242 & $-0,76$ \\
\hline A 2 & 440,91 & 392,65 & 486,67 & 0,00217 & 0,00193 & 0,00240 & $-0,74$ \\
\hline A 3 & 441,16 & 396,41 & 490,77 & 0,00217 & 0,00189 & 0,00241 & $-0,76$ \\
\hline A4 & 440,83 & 394,93 & 486,46 & 0,00217 & 0,00190 & 0,00244 & $-0,75$ \\
\hline A 5 & 440,90 & 397,38 & 486,36 & 0,00217 & 0,00194 & 0,00242 & $-0,76$ \\
\hline A6 & 441,06 & 392,78 & 494,68 & 0,00217 & 0,00191 & 0,00244 & $-0,75$ \\
\hline A 7 & 441,04 & 390,81 & 489,16 & 0,00217 & 0,00191 & 0,00241 & $-0,75$ \\
\hline A 8 & 440,78 & 395,22 & 494,89 & 0,00217 & 0,00188 & 0,00242 & $-0,75$ \\
\hline A9 & 441,25 & 395,50 & 484,87 & 0,00217 & 0,00194 & 0,00244 & $-0,74$ \\
\hline A 10 & 440,97 & 394,21 & 488,52 & 0,00217 & 0,00193 & 0,00240 & $-0,75$ \\
\hline Todas & 440,98 & 390,81 & 494,89 & 0,00217 & 0,00188 & 0,00244 & $-0,75$ \\
\hline
\end{tabular}




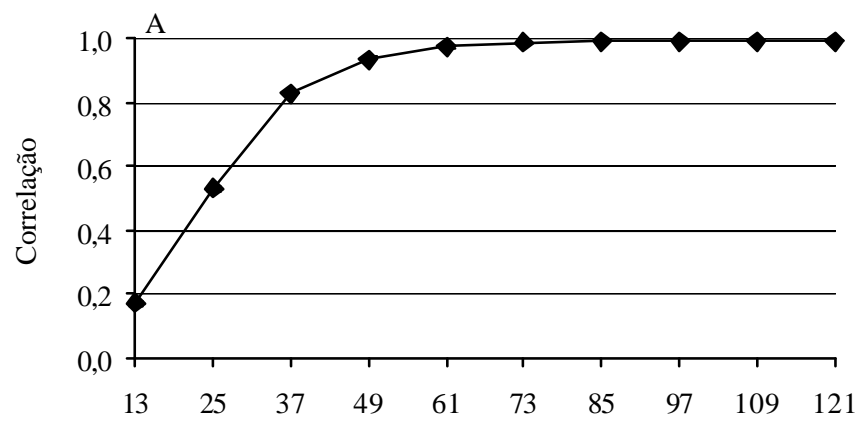

Número de pesagens

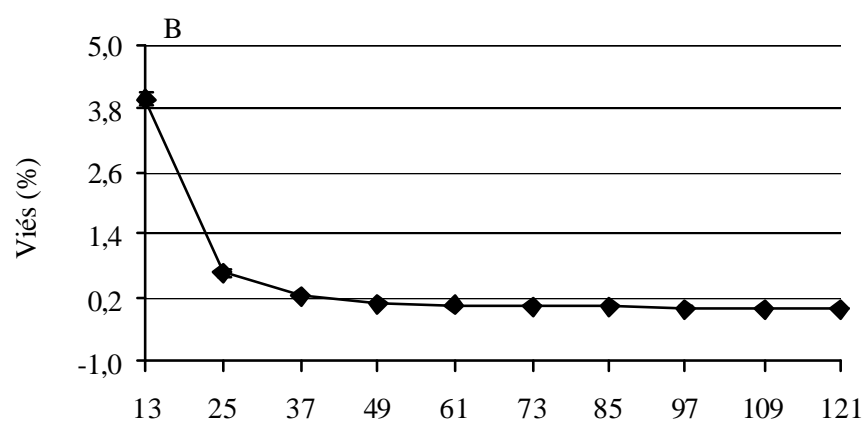

Número de pesagens

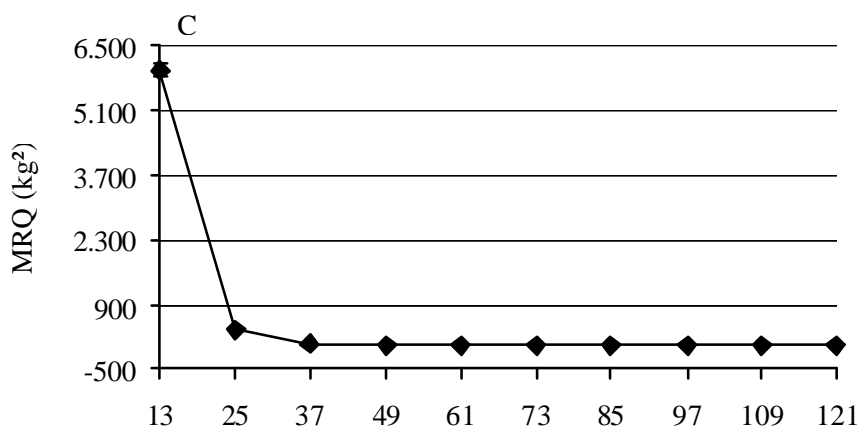

Número de pesagens

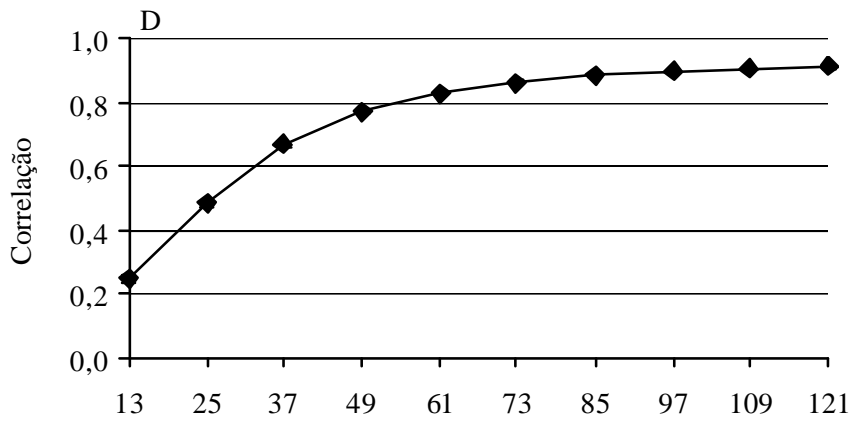

Número de pesagens

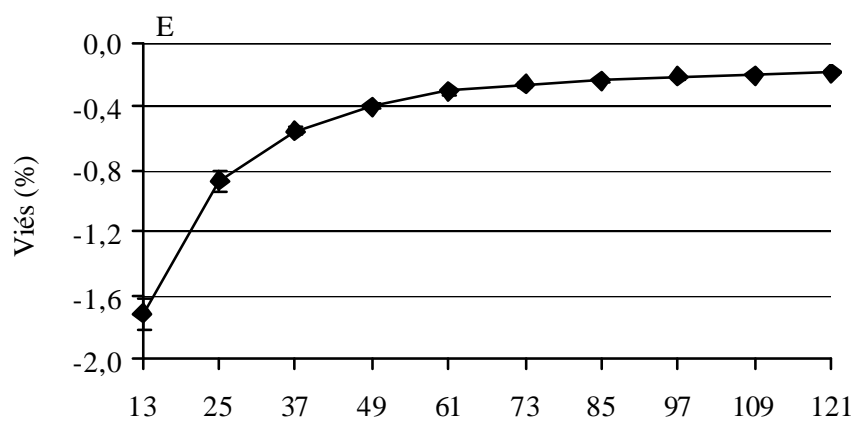

Número de pesagens

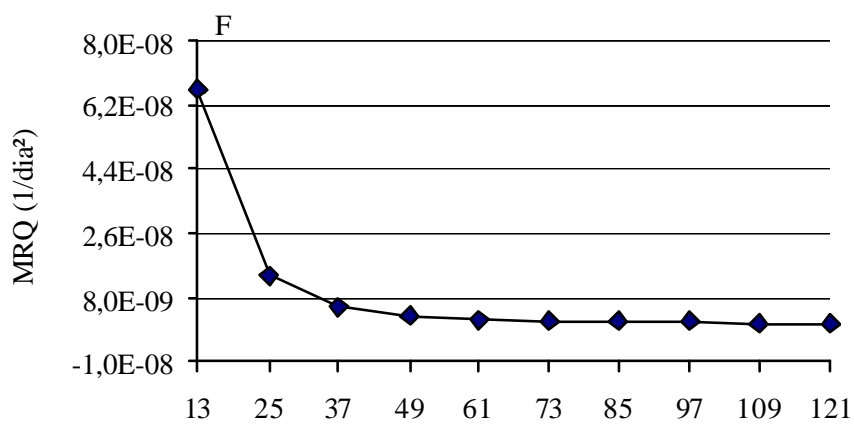

Número de pesagens

Figura 2 - Médias ( \pm desvios-padrão) das correlações ( $A$ e D), vieses (B e E) e médias dos resíduos ao quadrado (MRQ,C e F) das estimativas dos parâmetros $A$ (esquerda) e $k$ (direita) para os dez conjuntos de dados simulados, de acordo com o número de pesagens.

dos dois parâmetros pode ser explicada, parcialmente, pela correlação negativa existente entre eles. A redução do número de medidas utilizadas nas análises também provocou aumento das médias dos resíduos ao quadrado, especialmente quando o número de pesagens foi inferior a 37 .

A escassez de observações peso-idade em todo o ciclo de vida dos animais, especialmente a partir de 48 meses de idade, prejudica as estimativas dos parâmetros $A$ e $k$, concordando com as sugestões de Rosa et al. (2000). Dessa forma, se houver interesse na estimação de parâmetros do modelo de Richards (1959) para fêmeas bovinas pesadas mensalmente, recomenda-se que sejam utilizadas pesagens do nascimento aos 48 meses de idade, pelo menos.

As estatísticas para os parâmetros $A$ e $k$, de acordo com o intervalo de pesagens (Figura 3 ), foram obtidas considerando-se que todos os animais foram pesados até a idade de 3.601 dias, com alterações do intervalo de pesagens e, conseqüentemente, do número de pesagens.

Para o parâmetro $A$, os aumentos dos intervalos de pesagens provocaram pequenas alterações nas médias das correlações entre os valores simulados e estimados, vieses e médias dos resíduos ao quadrado. Esse resultado está de 

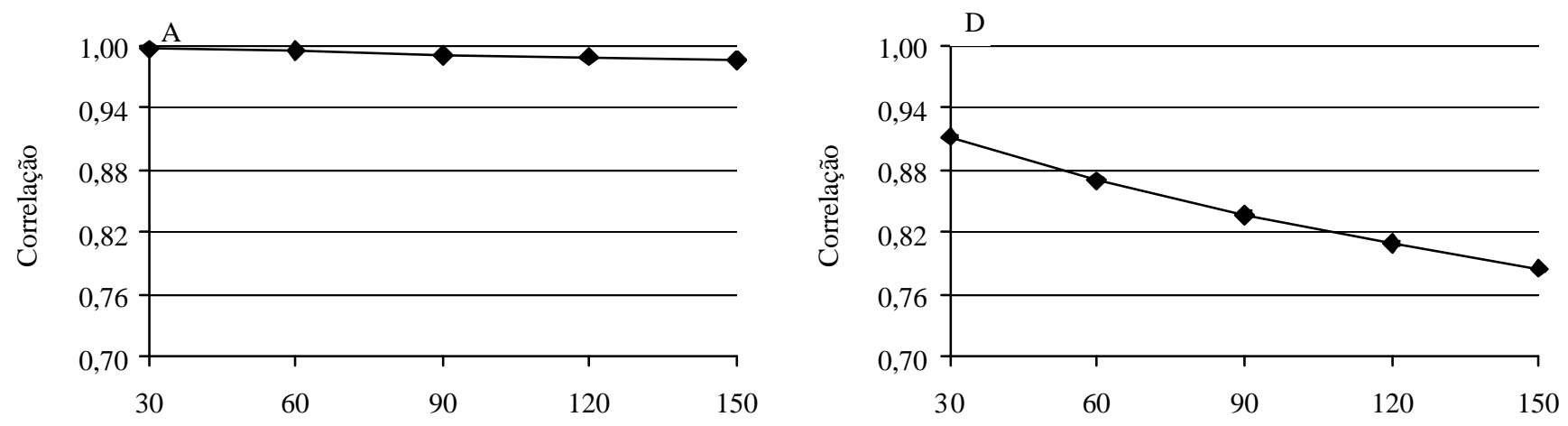

Intervalo de pesagens (dias)
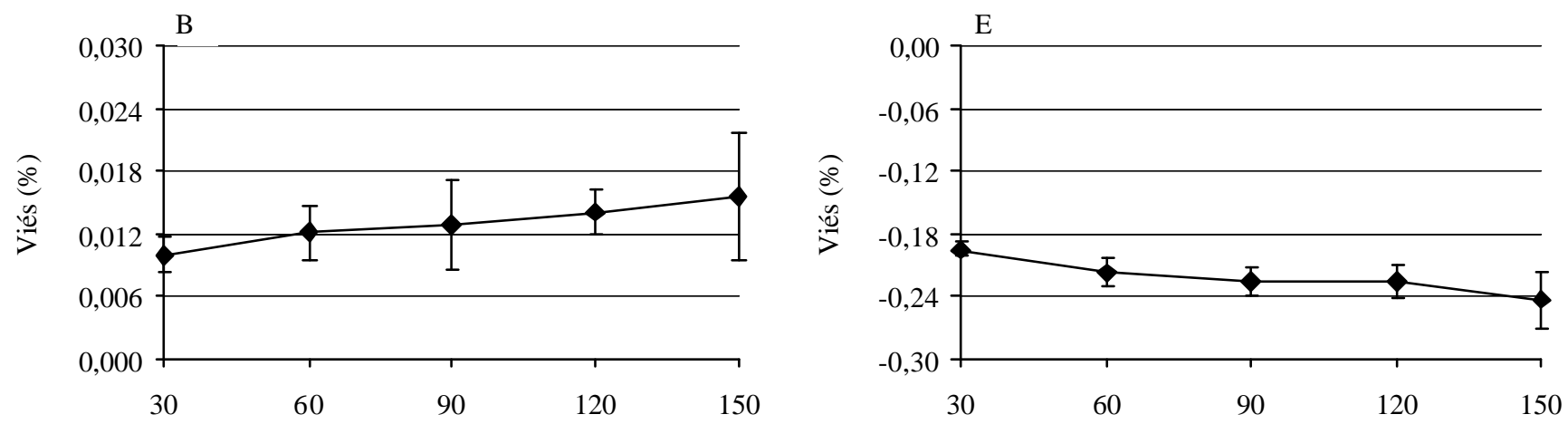

Intervalo de pesagens (dias)

Intervalo de pesagens (dias)
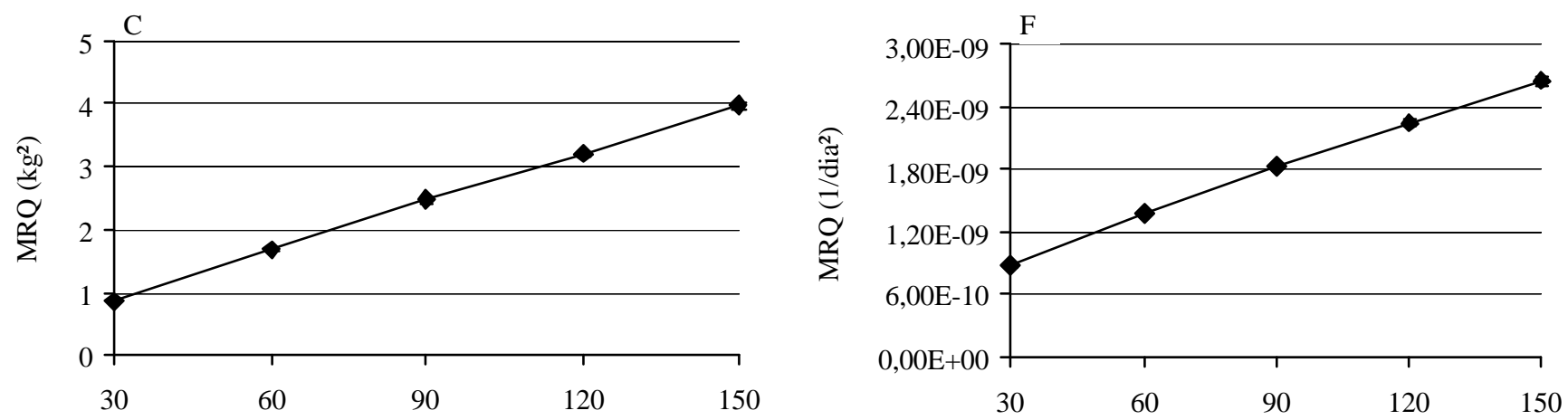

Intervalo de pesagens (dias)

Intervalo de pesagens (dias)

Figura 3 - Médias ( \pm desvios-padrão) das correlações ( $A$ e D), vieses (B e E) e médias dos resíduos ao quadrado (MRQ, C e F) das estimativas dos parâmetros $A$ (esquerda) e $k$ (direita) para os dez conjuntos de dados simulados, de acordo com o intervalo de pesagens.

acordo com as variações observadas nas estimativas dos parâmetros do modelo de Richards (1959) relatadas por Nobre et al. (1987). Esses autores, que trabalharam com o mesmo modelo para ajuste dos dados de crescimento de bovinos Nelore do nascimento aos 24 meses de idade, obtiveram diferenças inferiores a $0,5 \%$ entre as estimativas do parâmetro $A$, considerando intervalos de pesagens de 30, 60 ou 90 dias. Para a estimação do parâmetro $A$, é importante que medidas de peso em idades avançadas estejam disponíveis. Quando o animal atinge a idade adulta, as diferenças nos pesos obtidos a intervalos de tempo superiores a um mês são, possivelmente, pequenas (exceto quando a vaca estiver gestante), o que pode não influenciar nas estimativas do peso adulto com a utilização de modelos não-lineares.

Para o parâmetro $k$, reduções superiores a $10 \%$ nas correlações entre os valores estimados e os simulados, em relação às correlações obtidas com a utilização de intervalos 
mensais, foram observadas quando o intervalo de pesagens foi de 120 dias ou mais. Contudo, é importante ressaltar que as correlações entre valores simulados e estimados de $k$, obtidas com a utilização de pesagens mensais, foram ligeiramente superiores a 0,9 , enquanto esta correlação foi 0,87 ou 0,84 quando o intervalo de pesagens foi de 60 ou 90 dias, respectivamente. Nobre et al. (1987) observaram redução de $5 \%$ e aumento de $7 \%$ nas estimativas do parâmetro $k$ quando foram consideraram pesagens bimestrais ou trimes- trais, respectivamente, em relação à estimativa obtida com a utilização de pesagens mensais. Para a estimação do parâmetro $k$, os dados obtidos durante a fase inicial de crescimento também são importantes, pois neste período, o peso muda rapidamente com o avançar da idade e o aumento no intervalo de pesagens implica redução na precisão com que a mudança do peso (que não é linear) é avaliada, prejudicando as estimativas do parâmetro, como verificado neste trabalho. Dessa forma, se houver interesse

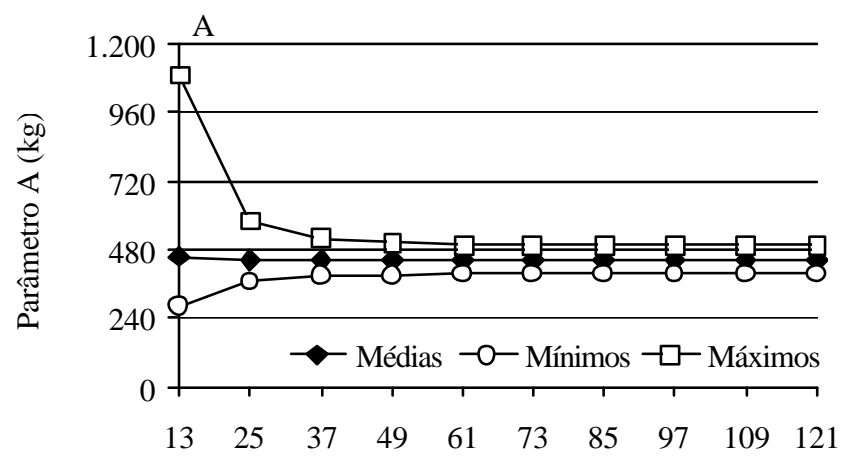

Número de pesagens

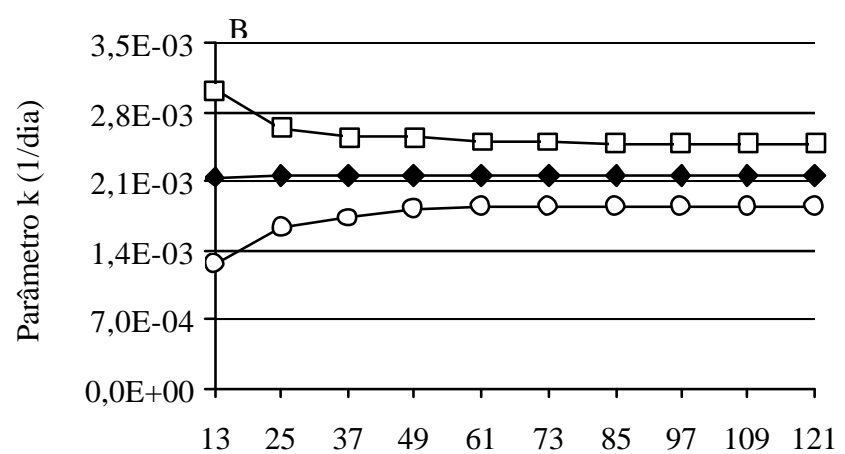

Número de pesagens

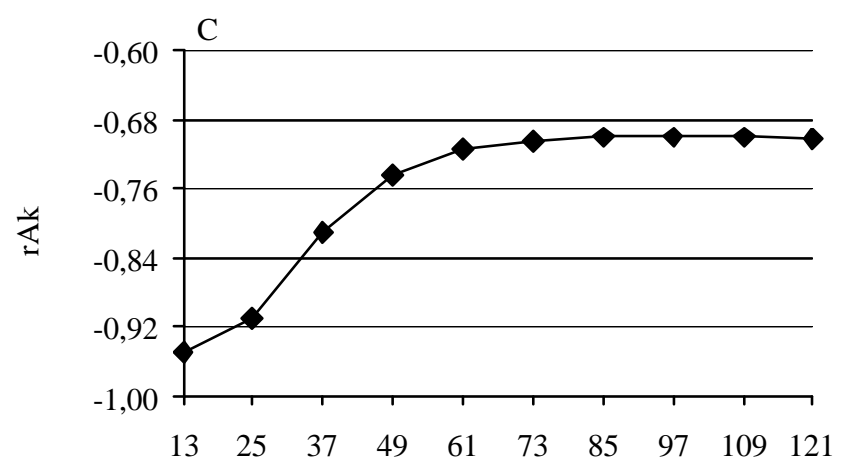

Número de pesagens

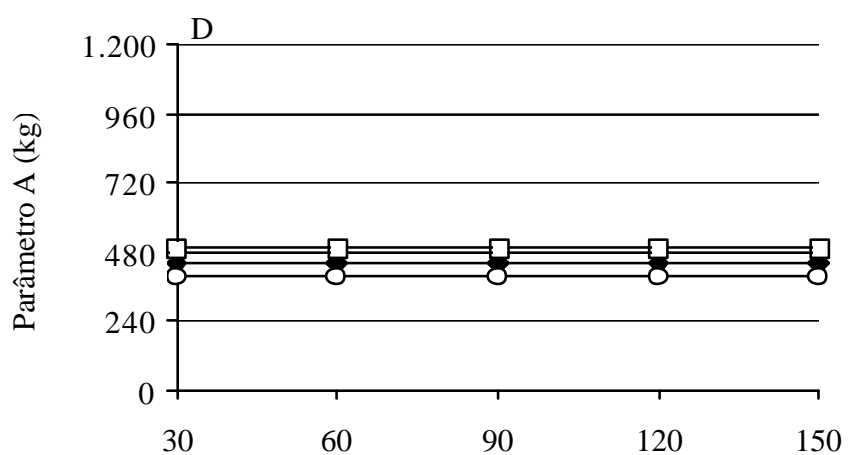

Intervalo de pesagens (dias)

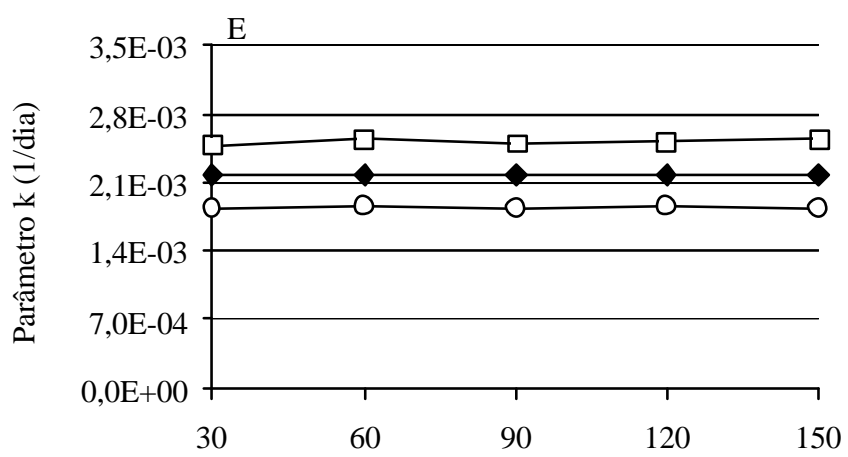

Intervalo de pesagens (dias)

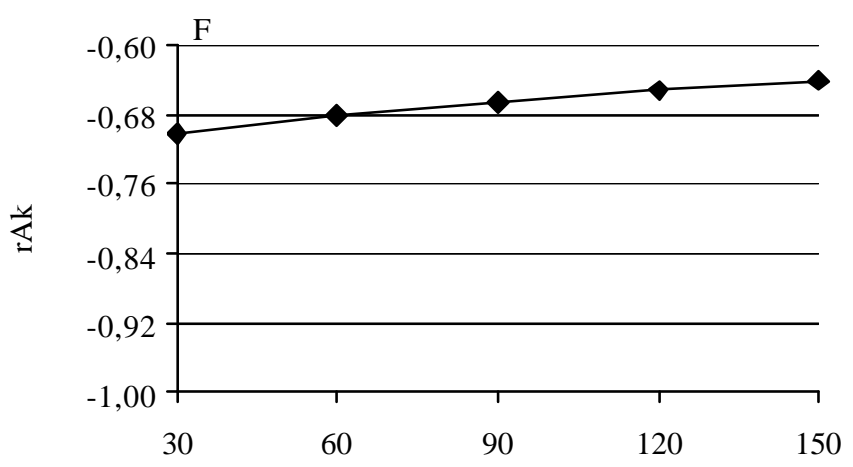

Intervalo de pesagens (dias)

Figura 4 - Médias, valores mínimos e máximos das estimativasdos parâmetros $A$ e $k$ e médias das correlações entre eles $\left(r_{A k}\right)$ para os dez conjuntos de dados simulados, de acordo com o número ( $A, B$ e $C$ ) e intervalo ( $D, E$ e $F)$ de pesagens. 
na comparação de taxas de maturação, estimadas a partir de modelos não-lineares com dados de fêmeas pesadas até idades próximas aos 10 anos, é importante que os pesos sejam avaliados em intervalos de tempo de 90 dias ou menos, caso contrário, podem ser obtidas estimativas não-representativas.

Em geral, as médias dos parâmetros $A$ e $k$ foram seme lhantes (Figura 4), independentemente do número ou do intervalo de pesagens considerados. Contudo, quando analisados os valores mínimos e máximos de $A$ e $k$ estimados a partir das análises com dados obtidos até a idade de 36 meses (37 observações com intervalos de 30 dias), as amplitudes observadas foram maiores que aquelas para os resultados das análises com pesagens mensais até 48 meses de idade, pelo menos (49, ou mais, medidas depesoidade obtidas mensalmente). Esses resultados corroboram aqueles obtidos por Oliveira et al. (1995) e Santoro et al. (2005), ambos utilizando o modelo não-linear de Richards (1959). Enquanto as estimativas de $A$ e $k$ obtidas por Oliveira et al. (1995) para fêmeas Guzerá pesadas até pelo menos 60 meses de idade variaram entre 347,48 e 787,75 kg e 0,0138 e $0,1359 /$ mês, respectivamente, aquelas obtidas por Santoro et al. (2005) para fêmeas Guzerá, Nelore e Nelore Mocho pesadas até no máximo 24 meses de idade variaram entre 214 e 991 kg, 201 e 998 kg e 205 e 956 kg (parâmetro A) e entre 0,000092 e 0,0051/dia, 0,000262 e 0,000669/dia e 0,000337 e 0,01041/dia (parâmetrok) para as raças Guzerá, Nelore e Nelore Mocho, respectivamente.

Os aumentos das amplitudes das estimativas de $A$ e $k$ podem ocasionar problemas estatísticos, pois, se as estimativas de $A$ e $k$ forem utilizadas para realização de análises de variância e testes de hipóteses, é possível que o aumento na amplitude leve ao aumento da probabilidade de ocorrência do erro tipo II (aceitar a hipótese de nulidade quando ela deve ser rejeitada). A existência de números diferentes de medidas peso-idade por animal também pode ocasionar heterogeneidade de variâncias residuais, uma vez que os animais com maior número de medidas teriam estimativas de $A$ e $k$ associadas a menores erros-padrão em relação àqueles animais com números inferiores de medidas.

Os aumentos dos intervalos de pesagens não proporcionaram aumentos nas amplitudes das estimativas de $A$ e $k$. Com a redução do número de pesagens obtidas mensalmente, a correlação entre $A$ e $k$ foi se aproximando de -1 , corroborando os resultados de Morrow et al. (1978). Com o aumento do intervalo de pesagens, as correlações foram se aproximando de 0 . A variação observada no último caso foi mais sutil que aquela observada no primeiro.

Após análise dos resultados apresentados anteriormente, foram gerados e analisados outros subarquivos de dados, nos quais foram consideradas as pesagens até $1.441,1.801$ ou 2.161 dias de idade (aproximadamente 4, 5 e 6 anos de idade, respectivamente) e com intervalos de pesagem de 30, 60, 90 e 120 dias. Assim, os números de pesagens disponíveis por animal foram de 49, 25, 17 e 13 para pesagens até 4 anos, 61, 31, 21 e 16 para pesagens até 5 anos e 73, 37, 25 e 19 para pesagens até 6 anos de idade, respectivamente.

As médias das correlações envolvendo as estimativas e os valores paramétricos de $A$ (Figura 5) foram 0,85 ou superiores, o que sugere boa confiabilidade quando os animais são pesados até 4 anos de idade, pelo menos.

Se os animais forem pesados até 4 anos de idade, é importante que os intervalos de pesagens não sejam superiores a 30 dias para evitar correlações entre as estimativas e os valores paramétricos de $k$ iguais ou inferiores a 0,70 (Figura 5). Essas correlações foram superiores a 0,75 quando foram combinados intervalo de pesagem de 60 dias com período de avaliação de 5 anos ou intervalo de 90 dias e avaliação por 6 anos (Figura 5).

Considerando aumento do período de pesagem de 4 para 5 ou 6 anos, houve redução dos critérios viés e média dos resíduos ao quadrado para $A$ e $k$ (Figura 5). Quando não for viável realizar pesagens mensais, é possível evitar correlações entre estimativas e valores paramétricos inferiores a 0,70 e reduzir o viés e a média dos resíduos ao quadrado com o aumento do período de avaliações.

Do ponto de vista prático, número e freqüência de pesagens menores poderiam reduzir a demanda de tempo e mão-de-obra, em comparação à pratica de pesagens mensais, sem perda de precisão nas medidas. Isso comprova a importância da determinação de número e intervalo de pesagens mínimos para a coleta de dados em experimentos sobre o crescimento de bovinos. Contudo, é importante ressaltar que o nível de precisão requerido pelo interessado nas estimativas influencia o número e o intervalo de pesagens que devem ser considerados. 


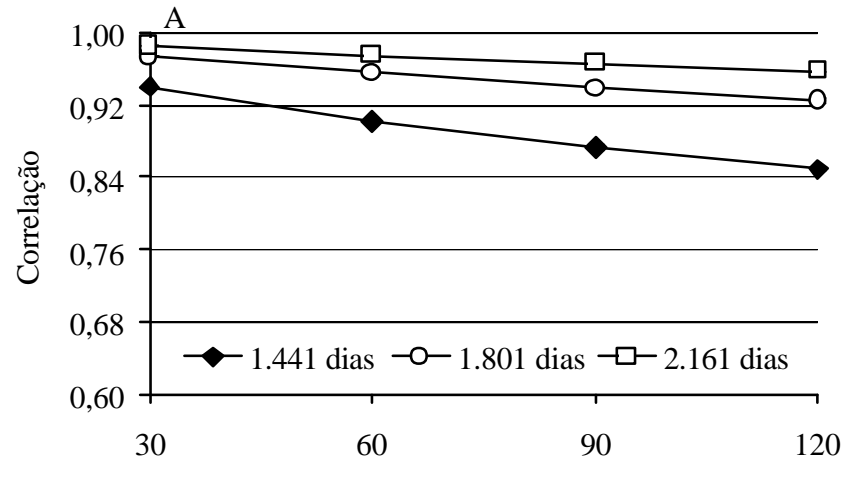

Intervalo de pesagens (dias)

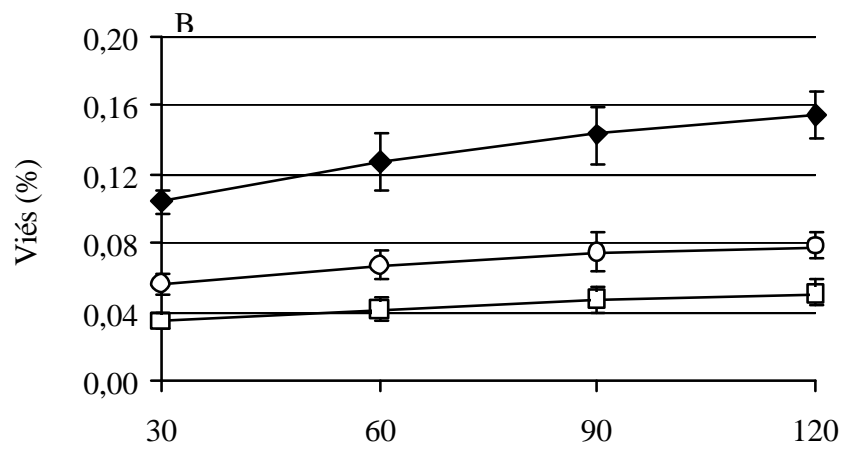

Intervalo de pesagens (dias)

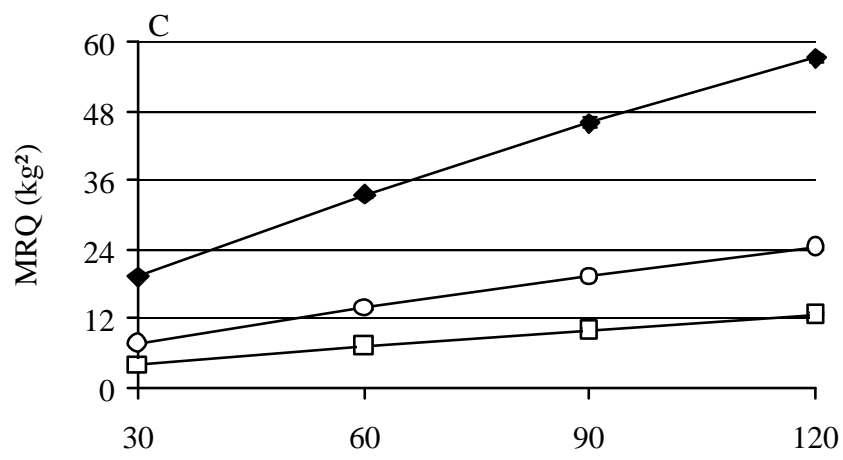

Intervalo de pesagens (dias)

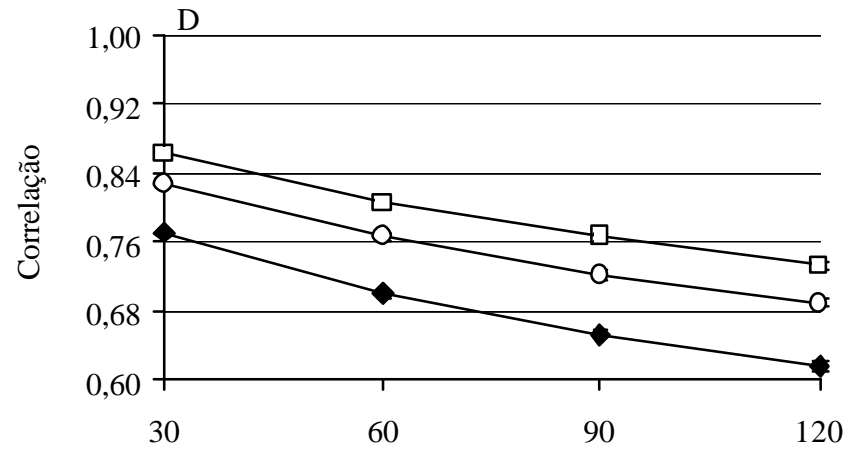

Intervalo de pesagens (dias)

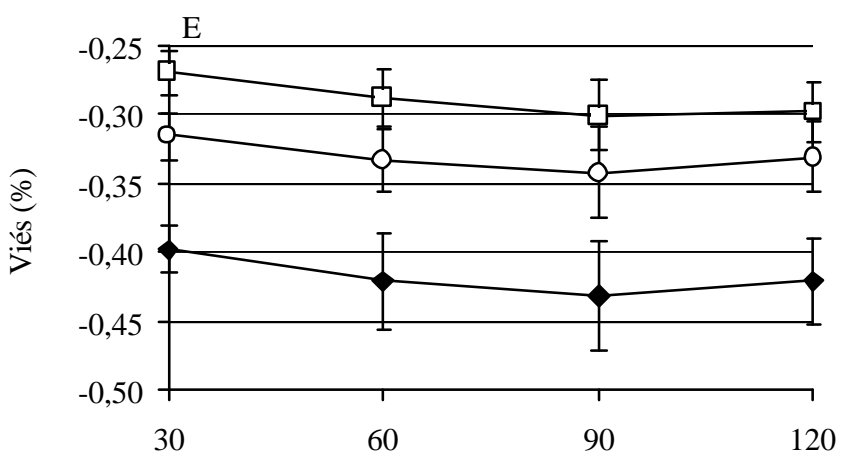

Intervalo de pesagens (dias)

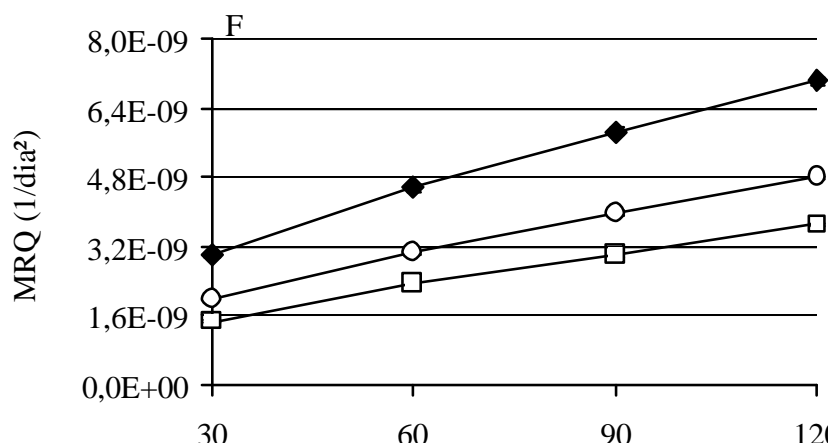

Intervalo de pesagens (dias)

Figura 5 - Médias ( \pm desvios-padrão) das correlações ( $A$ e D), vieses (B e E) e médias dos resíduos ao quadrado (MRQ, $C$ e $F$ ) das estimativas dos parâmetros $A$ (esquerda) e $k$ (direita) para os dez conjuntos de dados simulados, considerando pesos até as idade de $1.441,1.801$ e 2.161 dias e diferentes intervalos de pesagens.

\section{Conclusões}

O número e o intervalo de pesagens influenciam as estimativas dos parâmetros $A$ e $k$ do modelo não-linear de Richards. As estimativas de $A$ e $k$ se aproximam dos valores paramétricos à medida que diminui o intervalo e aumenta o período de pesagens. Maiores intervalos de pesagens podem ser combinados a maiores períodos de avaliação para obtenção de estimativa de $A$ e $k$ confiáveis. Assim, a utilização de medidas peso-idade, obtidas a cada
30 dias até 4 anos de idade, obtidas a cada 60 dias até 5 anos de idade ou obtidas a cada 90 dias até 6 anos de idade, são alternativas razoáveis quando há interesse na estimação do peso adulto e da taxa de maturação de fêmeas bovinas utilizando-se o modelo de Richards.

\section{Agradecimento}

Aos estudantes Emerson Alencar Bonelli, Fabiana Ferreira, Karine de Camargo, Leni Rodrigues Lima, Maryane 
Sespere Faria de Oliveira, Michelle Catiane Neves e Shirley Silva Carlesso, pelas sugestões durante a preparação do manuscrito. Este artigo é dedicado ao amigo Alessandro Luís Fraga (in memoriam).

\section{Literatura Citada}

BARBOSA, P.F.; ALENCAR, M.M.; SILVA, A.M. Peso à maturidade, taxa de maturação e eficiência produtiva em fêmeas da raça Canchim. Arquivo Brasileiro de Medicina Veterinária e Zootecnia, v.54, n.5, p.510-517, 2002.

BROWN, J.E.; FITZHUGH JR., H.A.; CARTWRIGHT, T.C. A comparison of nonlinear models for describing weight-age relationships in cattle. Journal of Animal Science, v.42, n.4, p.810-818, 1976.

CLÉMENT, V.; BIBÉ, B.; VERRIER, E. et al. Simulation analysis to test the influence of model adequacy and data structure on the estimation of genetic parameters for traits with direct and maternal effects. Genetic Selection Evolution, v.33, n.4, p.369-395, 2001.

COELHO, J.G.; BARBOSA, P.F.; SANCHES, A. et al. Correlações entre parâmetros da curva de crescimento e medidas de eficiência produtiva de fêmeas da raça Holandesa Preta e Branca. In: REUNIÃO ANUAL DA SOCIEDADE BRASILEIRA DE ZootecniA, 43., 2006, João Pessoa. Anais... João Pessoa: SBZ/UFPB, [2006]. (CD-ROM).

FERRELL, C.L.; JENKINS, T.G. Energy utilization by mature, nonpregnant, nonlactating cows of different types. Journal of Animal Science, v.58, n.1, p.235-243, 1984.

GARNERO, A.V.; MARCONDES, C.R.; BEZERRA, L.A.F. et al. Parâmetros genéticos da taxa de maturação e do peso assintótico de fêmeas da raça Nelore. Arquivo Brasileiro de Medicina Veterinária e Zootecnia, v.57, n.5, p.652-662, 2005.

LÓPEZ DE TORRE, G.; CANDOTTI, J.J.; REVERTER, A. et al. Effects of growth curve parameters on cow efficiency.Journal of Animal Science, v.70, n.9, p.2668-2672, 1992.

McLAREN, J.B.; MORROW, R.E.; BUTTS, W.T. Impact of number and frequency of weighings on bovine weight-age curve parameters. Journal of Animal Science, v.54, n.1, p. 51-57, 1982.
MORROW, R.E.; McLAREN, J.B.; BUTTS, W.T. Effect of age on estimates of bovine growth-curve parameters. Journal of Animal Science, v.47, n.2, p.352-357, 1978.

NOBRE, P.R.C.; ROSA, A.N.; SILVA, L.O.C. et al. Curvas de crescimento de gado Nelore ajustadas para diferentes freqüências de pesagens. Pesquisa Agropecuária Brasileira, v.22, n.9-10, p.1027-1037, 1987.

OLIVEIRA, H.N.; LÔBO, R.B.; PEREIRA, C.S. Comparação de modelos não-lineares para descrever o crescimento de fêmeas Guzerá. Pesquisa Agropecuária Brasileira, v.35, n.9, p.1843-1851, 2000.

PAZ, C.C.P; PACKER, I.U.; FREITAS, A.R. et al. Influência de polimorfismos genéticos sobre os parâmetros da curva de crescimento em bovinos de corte. Revista Brasileira de Zootecnia, v.33, n.4, p.858-869, 2004.

PEROTTO, D.; CASTANHO, M.J.P.; ROCHA, J.L. et al. Descrição das curvas de crescimento de fêmeas bovinas Guzerá, Gir, Holandês x Guzerá e Holandês x Gir. Revista Brasileira de Zootecnia, v.26, n.2, p.283-288, 1997.

RICHARDS, J.F. A flexible growth function for empirical use. Journal of Experimental Botany, v.10, n.29, p.290-300, 1959.

ROSA, A.N.; LÔBO, R.B.; OLIVEIRA, H.N. et al. Variabilidade genética do peso adulto de matrizes em um rebanho Nelore do Estado de São Paulo. Revista Brasileira de Zootecnia, v.29, n.6, p.1706-1711, 2000.

SANTORO, K.R.; BARBOSA, S.B.P.; BRASIL, L.H.A. et al. Estimativas de parâmetros de curvas de crescimento de bovinos Zebu, criados no Estado de Pernambuco. Revista Brasileira de Zootecnia, v.34, n.6, p.2262-2279, 2005 (supl.).

SILVA, A.M.; ALENCAR, M.M.; FREITAS, A.R. et al. Herdabilidades e correlações genéticas para peso e perímetro escrotal de machos e características reprodutivas e de crescimento de fêmeas, na raça Canchim. Revista Brasileira de Zootecnia, v.29, n.6, p.2223-2230, 2000 (supl.2).

STATISTICAL ANALYSIS SYSTEM - SAS.User's guide. Version 9.1 ed. Cary: SAS Institute, 2002. (CD-ROM).

TEDESCHI, L.O.; BOIN, C.; NARDON, R.F. et al. Estudo da curva de crescimento de animais da raça Guzerá e seus cruzamentos alimentados a pasto, com e sem suplementação. 1. Análise e seleção das funções não-lineares. Revista Brasileira de Zootecnia, v.29, n.2, p.630-637, 2000. 